\title{
Erythrodermia Ichtyosis Formis Congenital - Case Report
}

\author{
Ambarkova $\mathbf{V}^{1 *}$, Zisovska $\mathrm{E}^{2}$, Kalcev $\mathbf{G}^{\mathbf{3}}$, Misevski $\mathbf{J}^{4}$, Damevska $\mathrm{K}^{\mathbf{5}}$, Ismaili $\mathrm{B}^{\mathbf{6}}$ and Ambarkov $\mathbf{J}^{\mathbf{7}}$ \\ ${ }^{1}$ University St. Cyril and Methodius, Faculty of Dental Medicine, Skopje, Republic of North Macedonia \\ ${ }^{2}$ University Clinic for Gynecology and Obstetrics, Skopje, North Macedonia \\ ${ }^{3}$ University Goce Delcev-Shtip, Faculty of Medical Sciences, North Macedonia \\ ${ }^{4}$ Ministry of Health, Skopje, North Macedonia \\ ${ }^{5}$ Chief of Pediatric Dermatology, University Clinic of Dermatology, Faculty of Medicine, University "Ss Kiril and Metodij", Skopje, Republic of \\ Macedonia \\ ${ }^{6}$ Policlinic Dr Bashkim, Gostivar, Republic of North Macedonia \\ ${ }^{7}$ Student at Faculty of medicine, University Ss. Cyril and Methodius, Skopje, Republic of North Macedonia \\ *Corresponding Author: Ambarkova V, University St. Cyril and Methodius, Faculty of Dental Medicine, Skopje, Republic of North \\ Macedonia.
}

Received: July 19, 2019; Published: September 20, 2019

DOI: $10.31080 /$ ASDS.2019.03.0643

\begin{abstract}
Non-bullous congenital ichthyosi-form erythroderma is an autosomal recessive congenital keratinization disorder.

Materials and Methods: We present the only one registered case of congenital non-bullous ichthyosiform erythroderma in North Macedonia.

Our patient now is a 29-year old man from Gostivar. He was born with low birth weight and a low Apgar score (5/7). After birth, the skin was so cracked, that there were bleeding in certain places. Furthermore, the entire body, especially the head, was covered with yellow-green scales of varying size, which were firmly attached to the substrate. He does not provide information about a similar or same illness in the family. The ophthalmologist detected ectropion on the eyes, congenital chronic dacryocystitis, and conjunctivitis. His nails are very fragile. Often, pruritus occurs due to dryness of the skin. Meanwhile, flexion contractures on the 4th and 5th fingers of the right arm are noticed. In addition, they are with reduced function. Little is known about the oral manifestations of this disorder. Conclusion: The prognosis is variable. The disease has a strong impact on the quality of life due to altered physical appearance, problematic symptoms and treatment restrictions.
\end{abstract}

Keywords: Congenital; Recessive; Non-Bullous Congenital Ichthyosiform Erythroderma; Oral Manifestations of Itchthyosis

\section{Introduction}

An ichthyosis is a heterogeneous group of hereditary diseases and also acquired forms. In the group of nonsyndromic ichthyoses, also known as autosomal recessive congenital ichthyosis (ARCI): arlequin ichthyosis, lamellar ichthyosis (LI), and congenital ichthyosiform erythroderma are included [1]. In the group of keratinopathic ichthyosis, which are coursed by to keratin mutations, epidermolytic ichthyosis (EI) and superficial epidermolytic ichthyosis are included.

The name derives from the Greek word "ichthys" - fish and is a descriptive name for the group of genodermatoses with impaired keratinization, followed by dry skin and appearance of peels/ squids of varying size, shape, and color. It usually manifests itself from birth and lasts until the end of life, so that it does not affect 
life expectancy, except in extremely severe forms. Nonbullous congenital ichthyosiform erythroderma (NBCIE) is an autosomal recessive form of inherited ichthyosis. The incidence of this disorder is about 1 in 300,000 births. Clinically, NBCIE appears as generalized erythroderma with fine white scales that gradually replace the collodion membrane. Other associations include ectropion, eclabium especially in babies born with Harlequin type ichthyosis [2-4], scalp alopecia, decreased sweating with heat intolerance, and nail dystrophy.

Ichthyosis is a heterogeneous group of the disease, it originates from the Greek word ichthys-fish and is a descriptive name for the group of gonadermatoses with impaired keratinization, followed by dry skin and appearance of peels/squids of varying size, shape and color. Ichthyosis usually manifests itself from the birth and lasts until the end of life, so that it does not affect life expectancy, except in extremely severe forms of Ichthyosis. Symptoms are different in each patient, so that two patients with the same type of Ichthyosis have different symptoms.

\section{Classification}

Ichthyosis covers more than 20 diseases, which are classified in the following basic clinical forms.

Ichthyosis vulgaris

Synonyms: Ichthyosis simplex, Ichthyosis vulgaris dominans.

Ichthyosis X-Conjuncta

Synonyms: Ichthyosis vulgaris recessive, X-linked recessive ichthyosis.

Ichthyosis Lamellaris $[5,6]$

Synonyms: Erythrodermia Ichthyosiformis congenital non bullosa.

Ichthyosis Epidermolytica

Synonyms: Erythrodermia Ichthyosiformis congenital bullosa, Hyperkeratosis epidermolytica, Ichthyosis bullosa.

Harlequin Foetus

Synonyms: Ichthyosis congenital letalis

Erythrodermia ichtyosisformis congenital

The incidence of moderate to severe ichthyosis is between 200400 every year. More than 16,000 babies are born with some form of Ichthyosis annually, according to The Foundation for Ichthyosis and Related Skin Types, Inc. ${ }^{\circledR}$ (FIRST), but the true incidence of ichthyosis may be even greater.

The incidence of Erythrodermia ichtyosisformis congenital is $1 / 200,000-1 / 1,000,000$. The newborn is often covered with a membrane (a thin membrane that looks like a child is covered with transparent foil, the skin is strongly erythematous, easily fractures and creates conditions for infections. These babies are often born prematurely After the disappearance of the cellophane lining appears a picture of ichthyosiform erythroderma, with hulls on the body and face, and larger limbs of the limbs. There is an ectropion that can persist throughout life, as well as hyperkeratosis of the palms and feet to varying degrees. Corneal white plaques on the skin cause flexor contractures of the limbs. Fingers are underdeveloped, intolerance to heat, alopecia, nail dystrophy, hearing. Erythema migrans over time may be less pronounced, but desquamation persists.

Erythrodermia ichtyosisformis congenital is a genetic heterogeneous disease that occurs due to certain gene mutations. Inheritance is autosomal recessive. In terms of gender, race and age, we can say that it affects people of all ages, races and sex.

\section{Diagnosis}

Generally, the diagnosis of ichthyosis, including all types and subtypes, is based on: clinical picture, positive family history, pathohistological findings, and an electron microscopic finding. In certain types, prenatal diagnosis is also possible. Also, the possibility of genetic testing is also not ruled out. Doctors often use genetic testing in order to help them in treating and monitoring the patient. Another reason for the need for genetic testing is if a person who has ichthyosis or a family member plans to form a family.

\section{Monitoring and therapy}

Hereditary ichthyoses are life-threatening illnesses, and therapy is symptomatic in order to alleviate the symptoms. The treatment is aimed at reducing hyperkeratosis by means of keratolytics and hydration of dry skin with emollients. Local treatment is carried out with the application of preparations containing urea 5-10\% lotions or creams; salicylates $5-10 \%$ or combined with corticosteroids (cautiously in children at a young age due to danger of intoxication). Lacticum or lithic acid (citricum) 1-3\% is used in creams; vitamin A-acid 0,05\% (tretinoin). Corticosteroid creams are recommended for capturing the prevailing regions and erythrodermic forms. 
It is often necessary to swim in lukewarm water and skin care with neutral hydrating creams of vegetable origin and avoiding supposons or $\mathrm{pH} 7$.

In severe cases, systemic therapy with: corticosteroids at moderate doses of pednisolon is used temporarily. Oral retinoids (acitretinum-neotigason) can be used in severe forms of the disease, but are less tolerated than in the case of Lamellar Ichthyosis [7]. For some patients there is a marked improvement over time, but the disease often remains stable throughout life, with periods of exacerbation. The expected duration of life is normal. The disease has a strong impact on the quality of life due to altered physical appearance, troublesome symptoms and disease and therapy limitations.

\section{Methods and Materials}

We present the only one registered case of congenital nonbullous ichthyosiform erythroderma in North Macedonia.

\section{Case Report}

Our patient now is a 29-year old man from Gostivar. He was born with low birth weight and a low Apgar score (5/10). After birth, the skin was so cracked, that there were bleeding in certain places. Furthermore, the entire body, especially the head, was covered with yellow-green scales of varying size, which were firmly attached to the substrate. Otherwise, the pathohistological finding of a part of the skin of the thigh showed hyperkeratosis with focal parakeratosis. Stratum granulosum was present in places. Also, there was moderate acanthosis with preserved architectonics of the epidermis. In the upper dermis, there was an inflammatory infiltrate. Many sections of the blood vessels, hair follicles, and sweat glands in the dermis were seen. He does not provide information about a similar or same illness in the family. The ophthalmologist detected ectropion on the eyes, congenital chronic dacryocystitis, and conjunctivitis. His nails are very fragile. His nails do not grow upright but in a downward direction. Often, pruritus occurs due to dryness of the skin. Meanwhile, flexion contractures on the 4 th and 5th fingers of the right arm are noticed. In addition, they are with reduced function. Depressive symptoms like grief and sleep disturbance sometimes arise, due to its condition. Topical emollients and topical keratolytic are generally the mainstays of treatment.

When the patient was born, 29 years ago a biopsy from his skin was taken and sent to University of Zagreb for pathological analysis.

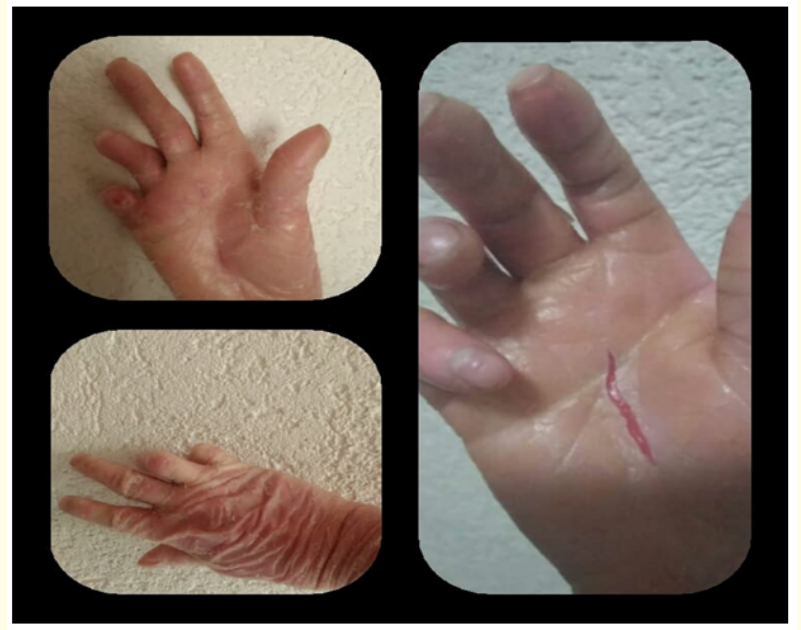

Figure 1: Clinical photos - Contractures on the fingers of patient's hands.

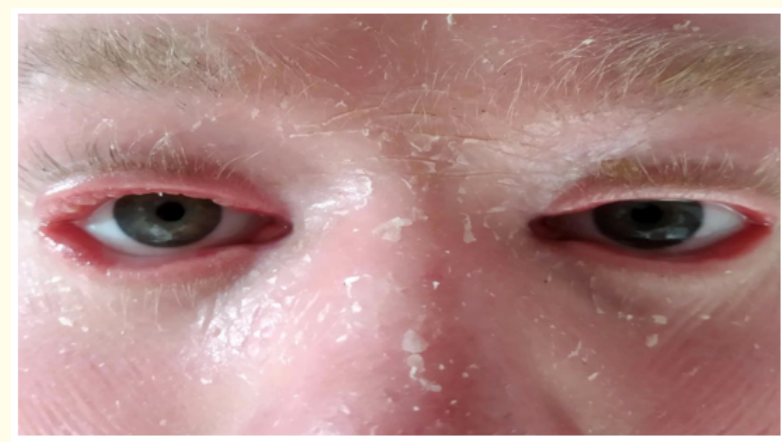

Figure 2: Ectropion of the both eyes.

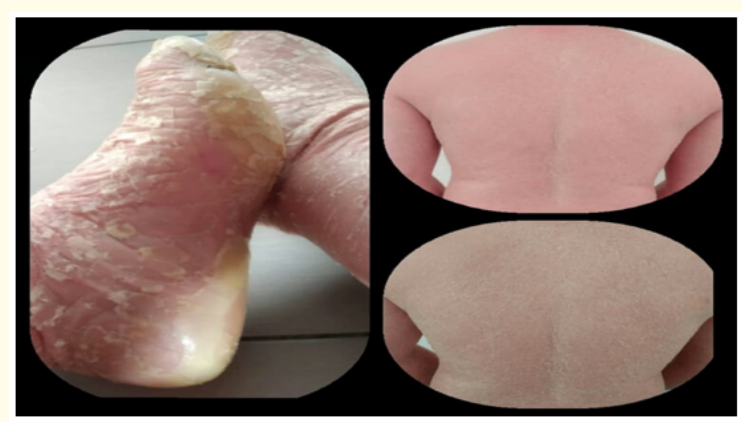

Figure 3: Palmoplantar keratoderma, contractures on the fingers of patient's legs on the left side of the picture. 


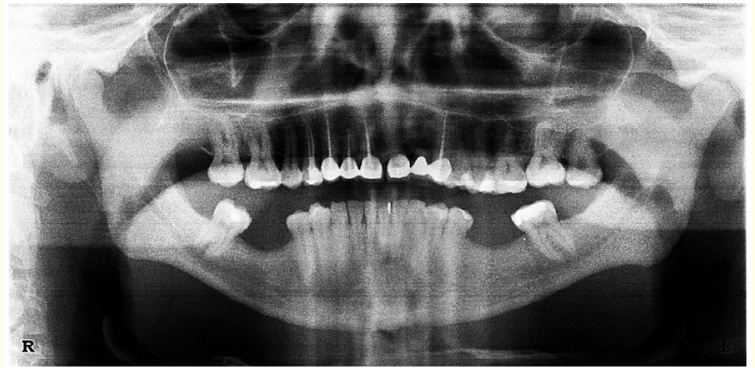

Figure 5: OPG of the Patient with Erythrodermia ichtyosis congenitalis

Radiological examination (figure 4) showed endodontic treatment at 11, 12, 13,14 and 23teeth,extraction of the 22 tooth and the presence of a fixed prosthodontics restoration, because missing tooth is only on one side of the mouth in the upper jaw.

The dental management was initiated with a preventive measures as the oral hygiene was compromised and patient was under high caries risk. Full mouth scaling and polishing was done followed by topical fluoride application.

For the management of his skin problem, he was referred to the Clinic of Dermatovenerology.

\section{Discussion}

Non-bullous congenital ichthyosiform erythroderma is characterized by grey-white scales on an erythematous background, induced by the accelerated mitotic rate of the epidermis and disruption of the epidermal barrier. Palmoplantar keratoderma, nail dystrophies, alopecia, ectropion, and anhidrosis may accompany the disease. Lamellar ichthyosis is another form of autosomal recessive non-bullous ichthyosis, which can be differentiated from NBCIE by the lack of erythroderma and the presence of characteristic large, dark-colored and plate-like scales. Recently, mutations in the genes ABCA12 [2,8-10] TGM1 [11], ALOXE3 [12], ALOX12B [13], NIPAL4 [14,15], and CYP4F22 [16] have been determined in cases with NBCIE and/or lamellar ichthyosis. Although these two disorders display several clinical and histopathological differences, they are assumed to fall on a continuum.

Mutation analysis in the study within 45 cases by Rajpopat S., et al. revealed that $52 \%$ of survivors had compound heterozygous mutations, whereas all deaths were associated with homozygous mutations. They concluded that with improved neonatal care and probably the early introduction of oral retinoids, the number of survivors is increasing. Also they concluded that compound heterozygotes appear to have a better survival advantage rate [17].

There is little knowledge and information about the oral manifestations of this disorder. Oral and dental findings reported in itchthyosis patients have included gingivitis, periodontitis, enamel hypoplasia, high caries incidence, delayed primary and secondary eruption, bruxism, bifid teeth, the irregular morphology of teeth, alveolar ridging, fish mouth appearance, mouth breathing, xerostomia, and hyperkeratotic plaques on the tongue $[1,6,18]$.

In the literature also case reports describing dental management of patients with NBCIE under general anesthesia could be found. One of such case report is described by Choudhary R., et al. threatening a 5 years and 11-month-old child with NBCIE suffering from early childhood caries (ECC) under general anesthesia [19].

In the literature, many case reports from different countries can be fined, described by different authors: Vergotine RJ [20], Ugezu CH described Harlequin ichthyosis [3], Nair KK described case report with lamellar ichthyosis [5], Ramar K described the oral manifestation of autosomal recessive congenital ichthyosis in a 2-year-old patient(1). Everyday new mutations associated with ichthyosis are presented in the literature [21,22]. Also Brown VL., et al. reported two NBCIE patients who have developed multiple aggressive nonmelanoma skin cancers, predominantly cutaneous squamous cell carcinoma. They climbed that NBCIE may be a risk factor for skin cancer development [23].

Glaucoma in patients with nonbullous congenital ichthyosiform erythroderma (NBCIE) is a rare entity. Ichhpujani P., et al. present a case of NBCIE with glaucoma and dwarfism. They concluded that the nonbullous congenital ichthyosiform erythroderma (NBCIE), glaucoma, and dwarfism can often occur together and need to be assessed and managed individually. Early diagnosis of this spectrum is very helpful for improvement of patient management and also for improvement of patient's quality of life. Dermatologists must cooperate with ophthalmologists and must get an ocular examination conducted for icthyoses patients [24].

As a side effects of oral retinoid therapy, angular cheilitis and facial dermatitis may occurs. 
Patents with ichthyosis do not require any modification in their dental treatment most of the times. However, as a pediatric dentists we should be aware of the concurrent medical problem and its treatment, and also about possibility of hepatic toxicity with the use of retinoids, which can affect the choice of local anesthetic during dental treatment.

During dental treatment care must be taken to avoid manipulating the patient's skin, particularly in the perioral areas, since affected areas can be tender or friable.

\section{Bibliography}

1. Ramar K., et al. "Oral manifestation of autosomal recessive congenital ichthyosis in a 2-year-old patient". Case Reports in Dentistry 2014 (2014): 483293.

2. Kelsell DP, Norgett EE, Unsworth H, Teh MT, Cullup T, Mein CA, Dopping-Hepenstal PJ, Dale BA, Tadini G, Fleckman P, Stephens KG, Sybert VP, Mallory SB, North BV, Witt DR, Sprecher E, Taylor AE, Ilchyshyn A, Kennedy CT, Goodyear H, Moss C, Paige D, Harper JI.

3. Ugezu CH., et al. "Harlequin Ichthyosis - A Case Report". Irish Medical Journal 110 (2017): 606.

4. Haftek M., et al. "A longitudinal study of a harlequin infant presenting clinically as non-bullous congenital ichthyosiform erythroderma". British Journal of Dermatology 135.3 (1996): 448-453.

5. Nair KK and Kodhandram G S. "Oral manifestations of lamellar ichthyosis: A rare case report”. Indian Journal of Paediatric Dermatology 17 (2016): 283-286.

6. Rathi NV., et al. "Oral manifestation of lamellar ichthyosis: A rare case report and review". Journal of Pakistam Association of Dermatologists 23.1 (2013): 99-102.

7. Damodaran K., et al. "A Unique Preparation and Delivery Method for Acitretin for Neonatal Harlequin Ichthyosis". Journal of Pediatric Pharmacology 23.2 (2018): 164-167.

8. Young BD., et al. "Mutations in ABCA12 underlie the severe congenital skin disease harlequin ichthyosis". American Journal of Human Genetics 76.5 (2005): 794-803.

9. Scott CA., et al. "Harlequin ichthyosis: ABCA12 mutations underlie defective lipid transport, reduced protease regulation and skin-barrier dysfunction". Cell and Tissue Research 351.2 (2013): 281-288.
10. Aggarwal S., et al. "Novel ABCA12 mutations in harlequin ichthyosis: a journey from photo diagnosis to prenatal diagnosis". Gene 556.2 (2015): 254-256.

11. Akiyama M., et al. "Novel mutations of TGM1 in a child with congenital ichthyosiform erythroderma". British Journal of Dermatology 144 (2001): 401-407.

12. Wang T., et al. "Homozygous ALOXE3 Nonsense Variant Identified in a Patient with Non-Bullous Congenital Ichthyosiform Erythroderma Complicated by Superimposed Bullous Majocchi's Granuloma: The Consequences of Skin Barrier Dysfunction". International Journal of Molecular Sciences 16.9 (2015): 21791-21801.

13. Victor F and Schaffer JV. "Lamellar ichthyosis". Dermatology Online Journal 11.4 (2005): 13.

14. Lin JC., et al. "Hyperlipidemia secondary to acitretin therapy for lamellar ichthyosis associated with a NIPAL4 mutation improves on a plant-based diet and relapses on a standard Western diet". Clinical Nutrition ESPEN 24 (2018): 54-57.

15. Simpson JK., et al. "Genotype-phenotype correlation in a large English cohort of autosomal recessive ichthyosis". British Journal of Dermatology 6 (2019).

16. Hotz A., et al. "Mutation update for CYP4F22 variants associated with autosomal recessive congenital ichthyosis". Human Mutation 39.10 (2018): 1305-1313.

17. Rajpopat S., et al. "Harlequin ichthyosis: a review of clinical and molecular findings in 45 cases". Archives of Dermatological Research 147.6 (2011): 681-686.

18. Ahn H and Yoon RK. "Netherton syndrome: dental considerations". Journal of Clinical Pediatric Dentistry 34.1 (2009): 7779.

19. Choudhary R and Satish V. "Dental Treatment of a Child Suffering from Non-bullous Congenital Ichthyosiform Erythroderma under General Anesthesia". International Journal of Clinical Pediatric Dentistry 8.2 (2015): 157-162.

20. Vergotine RJ., et al. "Harlequin ichthyosis: a case report". Pediatric Dentistry 35.7 (2013): 497-499.

21. E. Pohler., et al. "Novel autosomal dominant mutation in loricrin presenting as prominent ichthyosis". British Journal of Dermatology 173.5 (2015): 1291-1294. 
22. Hatsell SJ., et al. "Novel splice site mutation in keratin 1 underlies mild epidermolytic palmoplantar keratoderma in three kindreds". Journal of Investigative Dermatology 116.4 (2001): 606-609.

23. Brown VL., et al. "Multiple aggressive squamous skin cancers in association with nonbullous congenital ichthyosiform erythroderma". British Journal of Dermatology 158.5 (2008): 1125-1128.

24. Ichhpujani P., et al. "Juvenile Open Angle Glaucoma with Nonbullous Congenital Ichthyosiform Erythroderma". Journal of Glaucoma 27.11 (2018): e180-e182.

Volume 3 Issue 10 October 2019

(C) All rights are reserved by Ambarkova V., et al. 